\title{
LENGTH AND AREA ESTIMATES OF THE DERIVATIVES OF BOUNDED HOLOMORPHIC FUNCTIONS
}

\author{
SHINJI YAMASHITA
}

\begin{abstract}
MacGregor [1] and Yamashita [5] proved the estimates of the coefficient $a_{n}$ of the Taylor expansion $f(z)=a_{0}+a_{n} z^{n}+\cdots$ of $f$ nonconstant and holomorphic in $|z|<1$ in terms of the area of the image of $|z|<r<1$ by $f$ and the length of its outer or exact outer boundary. We shall consider some analogous estimates in terms of the non-Euclidean geometry for $f$ bounded, $|f|<1$, in $|z|<1$. For example, $2 \pi r^{n}\left|a_{n}\right| /\left(1-\left|a_{0}\right|^{2}\right)$ is strictly less than the non-Euclidean length of the boundary of the image of $|z|<r$, the multiplicity not being counted.
\end{abstract}

1. Introduction. Unless otherwise specified, by $f$ we always mean a function nonconstant, holomorphic, and bounded, $|f|<1$, in the disk $U=\{|z|<1\}$. The non-Euclidean metric in $U$ is expressed by the differential form $\mu(z)|d z|, \mu(z)=$ $\left(1-|z|^{2}\right)^{-1}, z \in U$. Then $\Delta(z, r)=\{w \in U ;|w-z| /|1-\bar{z} w|<r\}$ is the nonEuclidean disk of the non-Euclidean center $z \in U$ and the non-Euclidean radius $(1 / 2) \log [(1+r) /(1-r)](0<r<1)$.

By the image $g(G)$ of a domain $G$ by a function $g$ holomorphic in $G$ we mean the set of $w$ in the $w$-plane such that $w=g(z)$ for at least one $z \in G$; simply, $g(G)$ is the projection of the Riemannian image of $G$ by $g$. The exact outer boundary $C^{\sharp}(r, z)$ of $D(r, z) \equiv D(r, z, f)=f(\Delta(z, r))$ is the boundary of the unbounded component of the complement of the closure of $D(r, z)$ in the plane; see [5]. Roughly, $C^{\sharp}(r, z)$ is the boundary $\partial D(r, z)$ of $D(r, z)$ minus the "shorelines" of the "bays" and the "lakes" of the "island" $D(r, z)$. Furthermore, $C^{\sharp}(r, z)$ is a Jordan curve consisting of a finite number of analytic arcs. Let

$$
\lambda(r, z)=\int_{C^{\sharp}(r, z)} \mu(w)|d w|
$$

be the non-Euclidean length of $C^{\sharp}(r, z)$. The non-Euclidean length of $\partial D(r, z)$ is thus not smaller than $\lambda(r, z)>0$.

A non-Euclidean version of S. Yamashita's estimate [5, Theorem 2] is

THEOREM 1. Let $f$ be nonconstant, holomorphic, and bounded, $|f|<1$, in $U$. Let $n \equiv n(z)$ be the first number such that $f^{(n)}(z) \neq 0, n \geqslant 1, z \in U$. Then, for each $r$, $0<r<1$,

$$
\begin{gathered}
2 \pi r^{n}\left(1-|z|^{2}\right)^{n}\left|f^{(n)}(z)\right| /\left[n !\left(1-|f(z)|^{2}\right)\right] \\
\leqslant \Phi(\lambda(r, z))<\lambda(r, z),
\end{gathered}
$$

where, for $0 \leqslant x<+\infty, \Phi(x) \geqslant 0$ and $\Phi(x)^{2}=2 \pi\left(\pi^{2}+x^{2}\right)^{1 / 2}-2 \pi^{2}$.

Received by the editors March 24, 1982

1980 Mathematics Subject Classification. Primary 30C99; Secondary 30C50, 30C80.

(c) 1983 American Mathematical Society 0002-9939/82/0000-0971/ $\$ 02.00$ 
We note that $(0<) \Phi(x)<x$ for $x>0$, so that the second inequality in $(1.1)$ is immediate.

In particular, (1.1) for $z=0$, together with

$$
f(z)=a_{0}+a_{n} z^{n}+\cdots \quad\left(a_{n} \neq 0\right),
$$

yields that

$$
2 \pi r^{n}\left|a_{n}\right| /\left(1-\left|a_{0}\right|^{2}\right) \leqslant \Phi(\lambda(r, 0))<\lambda(r, 0)
$$

this is a non-Euclidean counterpart of [5, Theorem 1].

For the proof of Theorem 1 we shall make use of the following Theorem 2; unfortunately, the formulation appears to be complicated.

Let $E$ be the unbounded component of the complement of $D(r, z)$, not that of the closure of $D(r, z)$. Let $D^{\wedge}(r, z)$ be the complement of $E$. Then $D^{\wedge}(r, z)$ consists of the "island" $D(r, z)$ plus its reclaimed "lakes". As is pointed out by T. H. MacGregor [1, p. 319; 2, Lemma 2], the domain $D^{\wedge}(r, z)$ is simply connected whose boundary is called the outer boundary of $D(r, z)$. Thus, for $f(z)=(z+1 / \sqrt{3})^{3} / 8$, $D(r, 0)$ is a proper subset of $D^{\wedge}(r, 0)$ if $1 / 2<r<1$, while $D(r, 0)=\hat{D^{\wedge}}(r, 0)$ if $0<r \leqslant 1 / 2$.

Returning to our general $f$ we let

$$
\alpha(r, z) \equiv \alpha(r, z, f)=\iint_{\hat{D}(r, z)} \mu(w)^{2} d x d y \quad(w=x+i y)
$$

be the non-Euclidean area of $\hat{D}(r, z)(0<r<1, z \in U)$.

THEOREM 2. Let $f$ and $n(z)$ be as in Theorem 1 . Then, for each $r, 0<r<1$,

$$
\pi r^{2 n}\left(1-|z|^{2}\right)^{2 n}\left|f^{(n)}(z) /\left[n !\left(1-|f(z)|^{2}\right)\right]\right|^{2} \leqslant \alpha(r, z) .
$$

Specifically, (1.3) for $z=0$ with (1.2) reads

$$
\pi r^{2 n}\left[\left|a_{n}\right| /\left(1-\left|a_{0}\right|^{2}\right)\right]^{2} \leqslant \alpha(r, 0),
$$

for all $r, 0<r<1$. This is a non-Euclidean counterpart of MacGregor's estimate [1, Theorem 1]:

$$
\pi r^{2 n}\left|a_{n}\right|^{2} \leqslant a(r, 0)
$$

where $a(r, 0)$ is the Euclidean area of $D(r, 0)$, not that of $D^{\wedge}(r, 0)$ for $f$ of (1.2) not necessarily bounded in $U$. Since $a(r, 0) \leqslant \alpha(r, 0)$ for $|f|<1$, (1.4) yields a better estimate than (1.5) if $\left|a_{0}\right|$ is so near 1 that

$$
\left(1-\left|a_{0}\right|^{2}\right)^{2} \alpha(r, 0) \leqslant a(r, 0) .
$$

2. Proofs. In the proof of his theorem [1, Theorem 1] MacGregor makes use of the following improvement of [3, Theorem 4.7, p. 80].

MACGREGor's Lemma. Let

$$
g(z)=b_{0}+b_{n} z^{n}+\cdots \quad\left(b_{n} \neq 0, n \geqslant 1\right)
$$

be holomorphic in $U$, and let $r_{1}$ be the inner radius [3, p. 79] of $g(U)$ at $b_{0}$. Then

$$
\left|b_{n}\right| \leqslant r_{1} \text {. }
$$


Proof of Theorem 2. First we prove (1.4), then (1.3). For the proof of (1.4) we let

$$
g(z) \equiv f(r z)=a_{0}+a_{n} r^{n} z^{n}+\cdots \quad \text { in } U,
$$

and let $r_{1}$ be the inner radius of $g(U)=D(r, 0)$ at $a_{0}$. Then the estimate (2.1), together with $b_{n}=a_{n} r^{n}$, yields

$$
\left|a_{n}\right| r^{n} \leqslant r_{1}
$$

Let $r_{2}$ be the inner radius of $D^{\wedge}(r, 0)$ at $a_{0}$. Then $r_{1} \leqslant r_{2}$ because $D(r, 0) \subset D^{\wedge}(r, 0)$; see $[3$, p. 80].

Let $D^{*}$ be the circular symmetrization $\left[3\right.$, p. 69] of $D^{\wedge}(r, 0)$ with respect to the half-line $\left\{t a_{0} ; 0 \leqslant t<+\infty\right\}\left(=\{t ; 0 \leqslant t<+\infty\}\right.$, if $\left.a_{0}=0\right)$. Then $D^{*}$ is simply connected because the same is true of $D^{\wedge}(r, 0)$. Let $h(z)=a_{0}+c_{1} z+\cdots$ be a univalent holomorphic function in $U$ with $h(U)=D^{*}$. Then the inner radius $r_{3}$ of $D^{*}$ at $a_{0}$ satisfies $r_{3}=\left|c_{1}\right|\left[3\right.$, p. 79], and by [3, Theorem 4.8, p. 81], $r_{2} \leqslant r_{3}$, so that, by $(2.2)$,

$$
\left|a_{n}\right| r^{n} \leqslant r_{3}=\left|c_{1}\right|=\left|h^{\prime}(0)\right|
$$

whence follows

$$
r^{2 n}\left[\left|a_{n}\right| /\left(1-\left|a_{0}\right|^{2}\right)\right]^{2} \leqslant\left|h^{\prime}(0)\right|^{2} /\left(1-|h(0)|^{2}\right)^{2}
$$

because $h(0)=a_{0}$.

Since $\left|h^{\prime}\right|^{2} /\left(1-|h|^{2}\right)^{2}$ is subharmonic in $U$, and since the non-Euclidean area of $D^{*}$ is the same as that of $D^{\wedge}(r, 0)$, or, $\alpha(r, 0)$, it follows that

$$
\begin{aligned}
\left|h^{\prime}(0)\right|^{2} /\left(1-|h(0)|^{2}\right)^{2} & \leqslant(1 / \pi) \iint_{U}\left|h^{\prime}(z)\right|^{2} /\left(1-|h(z)|^{2}\right)^{2} d x d y \\
& =\alpha(r, 0) / \pi
\end{aligned}
$$

which, together with (2.3), yields (1.4).

To prove (1.3) we consider the composed function

$$
F(w)=f((w+z) /(1+\bar{z} w))
$$

of a variable $w \in U$. Since

$$
F^{(n)}(0) / n !=\left(1-|z|^{2}\right)^{n} f^{(n)}(z) / n !,
$$

$F(0)=f(z)$, and since $\alpha(r, 0, F)=\alpha(r, z, f),(1.3)$ is a consequence of (1.4) applied to $F$.

Proof of Theorem 1. The Gauss curvature $K(z)$ of the non-Euclidean space $U$ endowed with the metric $\mu(z)|d z|$ is given by

$$
K(z)=-4 \mu(z)^{-2}\left(\partial^{2} / \partial z \partial \bar{z}\right) \log \mu(z) \equiv-4 \text { in } U .
$$

Let $D^{\sharp}(r, z)$ be the domain bounded by the Jordan curve $C^{\sharp}(r, z)$. Then $D(r, z) \subset$ $\hat{D^{\prime}}(r, z) \subset D^{\sharp}(r, z)$. Let $A$ be the non-Euclidean area of $D^{\sharp}(r, z)$. Then

$$
\alpha(r, z) \leqslant A \text { and } 4 \pi A+4 A^{2} \leqslant \lambda(r, z)^{2}
$$


the latter is a consequence of $[4,(4.25)$, p. 1206], together with $K \equiv-4$. Consequently,

$$
2 \alpha(r, z) \leqslant\left(\pi^{2}+\lambda^{2}\right)^{1 / 2}-\pi .
$$

The estimate (1.1) now follows from (1.3) after a short computation.

3. Schwarz-Pick's lemma. As applications of Theorems 1 and 2 we obtain improvements of Schwarz-Pick's lemma:

$$
\left(1-|z|^{2}\right)\left|f^{\prime}(z)\right| /\left(1-|f(z)|^{2}\right) \leqslant 1, \quad z \in U .
$$

For example, let

$$
M(r, z)=\min (1, \Phi(\lambda(r, z)) /(2 \pi r)),
$$

for $0<r<1, z \in U$. If $f^{\prime}(z) \neq 0$, then we obtain by (1.1) that

$$
\left(1-|z|^{2}\right)\left|f^{\prime}(z)\right| /\left(1-|f(z)|^{2}\right) \leqslant M(r, z),
$$

while if $f^{\prime}(z)=0$, then the estimate is trivial. The estimate in terms of $\alpha(r, z)$ is similar, and is left as an exercise.

4. Concluding remarks. As to the sharpness of (1.4) on which (1.3) depends we have poor information: (1.4) is sharp in the limiting case, $r \rightarrow 0$. More precisely, let us be given $n \geqslant 1$ and $a_{0} \in D$. We set

$$
T(z)=\left(z+a_{0}\right) /\left(1+\overline{a_{0}} z\right)=a_{0}+b z+\cdots,
$$

and

$$
f(z)=T\left(z^{n}\right)=a_{0}+a_{n} z^{n}+\cdots,
$$

where $a_{n}=b$. Then, $\alpha(r, 0)=\alpha(r, 0, f)$ is the non-Euclidean area of $\Delta\left(a_{0}, r^{n}\right)$ which is the same as that of $\Delta\left(0, r^{n}\right)$, or $\alpha(r, 0)=\pi r^{2 n} /\left(1-r^{2 n}\right)$. Since

$$
\left|a_{n}\right| /\left(1-\left|a_{0}\right|^{2}\right)=|b| /\left(1-\left|a_{0}\right|^{2}\right)=1,
$$

(1.4) now reads

$$
\pi r^{2 n}=\pi r^{2 n}\left[\left|a_{n}\right| /\left(1-\left|a_{0}\right|^{2}\right)\right]^{2} \leqslant \pi r^{2 n} /\left(1-r^{2 n}\right),
$$

whence, the fact that $1-r^{2 n} \rightarrow 1$ as $r \rightarrow 0$ yields the sharpness in the limit.

Now, the situation explained at the end of $\S 1$ is obvious for the present $f$. For, given $0<r<1$, we choose a real $a_{0}$ so that

$$
0<a_{0}<1 \text { and }\left(1-a_{0}^{2} r^{2 n}\right)^{2}<1-r^{2 n} .
$$

A calculation shows that

$$
a(r, 0)=\pi r^{2 n}\left(1-a_{0}^{2}\right)^{2} /\left(1-a_{0}^{2} r^{2 n}\right)^{2},
$$

so that $\left(1-a_{0}^{2}\right)^{2} \alpha(r, 0)<a(r, 0)$.

Conversely, given a complex number $a_{0}$ with $1 / \sqrt{2}<\left|a_{0}\right|<1$, then for each $r$ with

$$
0<r<\left|a_{0}\right|<1 \text { and }\left(1-\left|a_{0}\right|^{2} r^{2 n}\right)^{2}<1-r^{2 n} \text {, }
$$


the same argument as above again shows that

$$
\left(1-\left|a_{0}\right|^{2}\right)^{2} \alpha(r, 0)<a(r, 0)
$$

\section{REFERENCES}

1. T. H. MacGregor, Length and area estimates for analytic functions, Michigan Math. J. 11 (1964), $317-320$.

2. Translations of the image domains of analytic functions, Proc. Amer. Math. Soc. 16 (1965), 1280-1286.

3. W. K. Hayman, Multivalent functions, Cambridge Univ. Press, London, 1967.

4. R. Osserman. The isoperimetric inequality, Bull. Amer. Math. Soc. 84 (1978), 1182-1238.

5. S. Yamashita, Length estimates for holomorphic functions, Proc. Amer. Math. Soc. 81 (1981), 250-252.

Tokyo Metropolitan University, Fukazawa, Setagaya-Ku, Tokyo (158), Japan 